\title{
IMOBILIZAÇÃO INTERNA DA COLUNA VERTEBRAL LOMBAR COM PLACAS DE CLORETO DE POLIVINILA, EM CÃES - PARTE I: RESULTADOS CLÍNICO-CIRÚRGICOS ${ }^{1}$
}

\author{
INTERNAL IMMOBILIZATION OF THE LUMBAR CANINE SPINE USING \\ POLYVINYLCHLORIDE PLATES - PART I: CLINICAL AND SURGICAL RESULTS
}

\section{Eduardo Alberto Tudury ${ }^{2}$ Cleuza Maria de Faria Rezende ${ }^{3}$ Leonardo Maciel Andrade $^{4}$}

\section{RESUMO}

Utilizando-se 20 cães, com o segmento lombar da coluna vertebral cirurgicamente desestabilizado na junção das vértebras LrL, foi testada a eficiência de placas ortopédicas de cloreto de polivinila (PVC) Tigre*, fixadas com parafusos nos corpos vertebrais e com fio ortopédico de aço inoxidável nos processos espinhosos. As placas foram manual e individualmente confeccionadas para cada animal e esterilizadas pelo calor. Apesar de apresentar maleabilidade adequada, a resistência das placas utilizadas no corpos vertebrais deixou a desejar, dado seu rompimento em $40 \%$ dos casos. Apesar disto, durante os 45 dias do estudo, verificaram-se resultados favoráveis quanto ao estado clinico-neurológico, resultados de exames laboratoriais, achados radiológicos, estabilidade e alinhamento da coluna vertebral, assim como, quanto a resistência e flexibilidade das placas utilizadas nos processos espinhosos. Os fios ortopédicos de aço inoxidável, utilizados para fixar as placas no compartimento dorsal da coluna vertebral, passaram entre e não através desses processos.

Palavras-chave: doenças da coluna vertebral, cirurgia no cão, tratamento defraturas, doenças do cão. Cloreto de polivinila, $P V C$.

\section{SUMMARY}

The efficiency ofpolyvinylchioride (PVC) orthopedic plates, fixed by screws to the vertebral bodies and by cerciage wires to the spinous processes, were tested in twenty dogs with the lumbar spine destabilized at $L ;-L]$. The plates were produced manually and individuatly for each animal and sterilized by heat. The plates exhibited suitable malleabitity but inappropriate strength because $40 \%$ ofthose affixed to the vertebral bodies were bmken. Nevertheless, during the 45 days ofthe study, the technique was consideredefective based on stability, clinicai, neurological, laboratory and radiological results. This efficiency was achieved by the technique used $m$ the spinous processes, where the plates were fixed by wires passing between (not through) them.

Key words: spinal diseases, surgery ofdogs, fracture fixation, dog diseases, Polyvinylchioride, PVC.

\section{INTRODUÇÃO}

As fraturas e luxações da coluna vertebral são vistas com frequência na clínica de pequenos animais (SMITH \& WALTER, 1985), devido principalmente a atropelamentos (MC. KEE, 1990). As lesões localizam-se comumente na região lombar (TURNER, 1987) e, como parte do tratamento, são indicadas as imobilizações internas da coluna vertebral (SWAIM, 1987; RENEGAR, 1990; BRUECKER \& SEIM, 1993). O emprego de placas, nos corpos vertebrais, impede a atuação de forças compressivas axiais e de cizalhamento, oferecendo adequada estabilidade rotacional e angular, diminuindo assim, as

\footnotetext{
${ }^{1}$ Parte da Tese apresentada pelo primeiro autor ao Curso de Doutorado em Ciência Animal da Escola de Veterinária da Universidade Federal de Minas Gerais (UFMG)

${ }^{2}$ Médico Veterinário, Doutor, Professor Adjunto 3, Departamento de Clínicas Veterinárias, Universidade Estadual de Londrina, Campus Universitário, Caixa Postal 6001, 86051-970, Londrina - PR. Autor para correspondência.

${ }^{3}$ Médico Veterinário, Doutor, Professor Adjunto 4, Departamento de Clínica e Cirurgia Veterinários, Escola de Veterinária, UFMG.

${ }^{4}$ Médico Veterinário, Mestrando da Escola de Veterinário, UFMG.
} 
possibilidades de falha do implante no pósoperatório (HOERLEIN, 1978; SMITH \& WALTER, 1985; SWAIM, 1987). A imobilização da coluna vertebral lombar mediante materiais rígidos fixados somente nos processos espinhosos dorsais, não é apropriada, mesmo em cães de pequeno porte (TUDURY et al., 1985). Dependendo do método pelo qual as placas são fixadas nesses processos, são observadas fraturas ou osteoporose dos mesmos (BRASMER \& LUMB, 1972; RISCHEN et al., 1987), assim como arrancamento de parafusos, deslizamento dorsal das placas e reações seromatosas (SMITH \& WALTER, 1985; WALKER tía/., 1985b; WALKER, 1990). Na espécie canina e com relação às fraturas vertebrais, ainda não existem estudos conclusivos sobre as forças mais importantes a serem anuladas pelo ato cirúrgico e no período pós-operatório (WALDRON et al., 1991). Entretanto, consideram-se as forças de flexão como as mais importantes na génese das injúrias espinhais e como causa das falhas de imobilização (WALTER $\boldsymbol{e t} \boldsymbol{a l}$., 1986).

As placas existentes para a imobilização da coluna vertebral de cães (WALKER, 1990; BRUECKER \& SEIM, 1993) não se encontram à disposição para compra no Brasil. Sua importação é possível, porém toma extremamente onerosa a imobilização interna da coluna vertebral. No país, são fabricadas e vendidas placas metálicas de aço inoxidável, específicas para a imobilização interna das fraturas de ossos longos de seres humanos. Mas, seu formato, espessura, tamanho e preço as tornam impróprias para o emprego em neurocirurgia de pequenos animais. BRASMER (1984) e SWAIM (1987) consideram as placas Lubra superiores às metálicas, para a fixação dos processos espinhosos. Por serem plásticas, as placas de polivinilideno podem ser facilmente cortadas e moldadas durante a cirurgia (YTURRASPE \& LUMB, 1972; RENEGAR, 1990), além de propiciarem sua fixação, nos processos espinhosos por fio de cerciagem, substituindo os parafusos (HOERLEIN, 1978; MÜLLER, 1991). A fixação de placas plásticas, simultaneamente, nos corpos e nos processos espinhosos da coluna vertebral lombar, é sugerida por YTURRASPE \& LUMB (1973) e WALKER et al., (1985b).

No Brasil, são utilizadas placas de cloreto de polivinila (PVC), na imobilização interna de fraturas de ossos longos de cães. (ALMEIDA \& REZENDE, 1990; REZENDE \& ALMEIDA, 1990; RODRIGUEZ ALVAREZ, 1990). Além do sucesso cirúrgico, as placas são mais viáveis economicamente, com a vantagem de poderem ser confeccionadas sob medida, para o osso a ser operado. Estas vantagens, poderiam se repetir quando da utilização destas placas na fixação de colunas vertebrais traumatizadas. RODRIGUEZ ALVAREZ (1990) utilizando as placas de PVC na imobilização de fraturas do terço distai do rádio e da uina de cães com idade inferior a 3 meses, cita que estas placas são uma alternativa económica e recomendável na prática ortopédica veterinária, apesar de observar ruptura da placa, reação periosteal e seroma.

O cloreto de polivinila é um plástico rígido, originado da polimerização do monômero cloreto de vinila. Estabilizantes, lubrificantes, modificadores de impacto, pigmentos, melhoradores de brilho, recheios e plastifícantes podem também ser agregados, para facilitar sua extrusão e melhorar sua apresentação e propriedades físicas (VINYL CHLORIDE, 1979; GOTTESMAN \& GOODMAN, 1985). Materiais de uso médico, feitos de PVC, como sondas, catéteres, contraceptivos, drenos, tubos dializadores, cânulas ruminais e intestinais, tampões esofágicos, próteses buço dentárias e placas ortopédicas (JANISH et al., 1972; SABADEANU $\boldsymbol{e t}$ al., 1972; HAYHURST \& WYMAN, 1975; WALKER et al., 1985a; MILLER\& WALTBY, 1986; KEMP \& KRUGER, 1987; CERVERA et al., 1989; REZENDE \& ALMEIDA, 1990; RODRIGUEZ ALVAREZ, 1990; GERHARDS et al., 1991; VASILEV et al., 1991; HUIBREGTSE, 1993; PAWDE \& MARUDWAR, 1993) permanecem no organismo de pacientes humanos e animais por períodos de dias até 18 meses.

RODRIGUEZ ALVAREZ (1 990) menciona que para a esterilização do PVC, pode-se usar a autoclave. KEMP \& KRUGER (1987) usam a fervura para a esterilização deste plástico. GOWARIKER et al. (1986) citam que o PVC, quando não aditivado deteriora-se sob temperaturas superiores a $200^{\circ} \mathrm{C}$. Quando aditivado com estabilizantes, o PVC resiste a temperatura de até $225^{\circ} \mathrm{C}$ (WILLIAMSOM \& KAVANAGH, 1987). As mudanças de cor do PVC, para o amarelo ou marrom, são indicativas de sua deterioração pelo calor (GOTTESMAN \& GODMAN, 1985).

Com relação aos cães, não há relatos na literatura que indiquem a ocorrência de tumores induzidos pelo PVC ou pelo cloreto de vinila. Caninos recebendo PVC, por via oral, junto com o alimento, além de ocasionais fezes amolecidas, não exibem alterações clínicas, nos exames sanguíneos, de necropsia ou histológicas dos órgãos (JOHNSON \& SCHMIDT, 1977). Após a exposição de cães ao cloreto de vinila, pela via aérea, em concentrações de 200 ppm, sete horas por dia, durante seis meses, não se observa alteração com relação ao peso corporal; peso 
do pulmão, coração, rim, baço e testículos; hemograma; FA; ALT; AST; ureia; urinálise e histopatologia hepática. Os trabalhos de FIGGE et al. (1978); KONTOMINAS et al. (1985) e DEMERTZIS \& KONTOMINAS (1986) assinalam que, tanto o cloreto de vinila e os plastifícantes, quanto os estabilizantes, podem ser eliminados ou diminuídos em concentração, se o plástico, antes de ser utilizado, for lavado com água e detergente, deixado em local ventilado por 14 dias e submerso sucessivamente em água destilada, álcool e óleo de oliva (não conservados em recipientes de PVC), por mais de quatro horas.

Neste trabalho são apresentados os resultados de observações clínico-cirúrgicas relacionados à eficiência das placas ortopédicas de PVC, na imobilização interna da coluna vertebral lombar desestabilizada de cães.

\section{MATERIAIS E MÉTODOS}

\section{Preparo das placas}

Para a confecção das placas foram utilizados tubos de PVC-Tigre ${ }^{\prime}$ ', de 4,8 e 10,2cm de diâmetro, de cor branca, com espessura de parede de 5 e $2 \mathrm{~mm}$, respectivamente. O PVC de $5 \mathrm{~mm}$ foi utilizado para a confecção das placas para os corpos vertebrais e o de $2 \mathrm{~mm}$ para as dos processos espinhosos. As placas dos corpos vertebrais tiveram seu comprimento estabelecido em $2 \mathrm{~mm}$ inferior à distância compreendida entre as extremidades cranial e caudal das vértebras lombar $2\left(\mathrm{~L}_{2}\right)$ e lombar $3\left(\mathrm{~L}_{3}\right)$, respectivamente. Sua largura foi igual à altura do corpo vertebral de $\mathrm{L}_{2}$ e oscilou entre 8 e $12 \mathrm{~mm}$. As placas a serem fixadas nos processos espinhosos foram confeccionadas aos pares e cortadas em arco, para melhor se adaptar à curvatura normal da coluna lombar. Sua largura oscilou entre 7 e $10 \mathrm{~mm}$ e seu comprimento foi $1 \mathrm{~cm}$ superior à distância compreendida entre as bordas cranial e caudal dos processos espinhosos das vértebras lombar $1\left(\mathrm{~L}_{1}\right)$ e lombar $4\left(\mathrm{~L}_{4}\right)$, respectivamente. A largura, como as outras medidas, foi determinada radiografícamente, de forma que as placas coubessem no espaço entre as lâminas vertebrais e o ligamento supraespinhal. Todas as placas foram confeccionadas, previamente à cirurgia e eram específicas para cada animal. Após estabelecer radiográfica e individualmente, as medidas e as formas, as placas foram delineadas com lápis nos tubos e cortadas utilizando-se serra elétrica tipo ticotico, dotada de lâmina para aço. Um orifício com diâmetro de Imm foi perfurado a $3 \mathrm{~mm}$ das extremidades das placas arciformes, com furadeira elétrica e brocas para aço. Com estes mesmos instrumentos, fizeram-se quatro orifícios nas placas para os corpos vertebrais $\mathrm{L}_{2}-\mathrm{L}_{3}$, com diâmetro de 4,70mm, para aquelas com largura igual a $12 \mathrm{~mm}$ e de $3,75 \mathrm{~mm}$, para as restantes. Estes orifícios, equidistantes no sentido dorsoventral e situados a 4 ou $5 \mathrm{~mm}$ de distância das extremidades das placas e do espaço intervertebral, foram dispostos de maneira que pudessem ser colocados dois parafusos em cada corpo vertebral,. Posteriormente a isto, todas as placas foram polidas manualmente, até obter-se bordas discretamente arredondadas e superfície lisa. Em seguida, foram lavadas e imersas sucessivamente (período de 12 horas) em água destilada, álcool etílico e azeite de oliva (não acondicionados em recipientes de PVC), e posteriormente, lavadas, enxaguadas, secadas e esterilizadas. A esterilização foi realizada por calor seco, à temperatura de $120^{\circ} \mathrm{C}$, por 60 minutos.

\section{Procedimentos cifnico-cirúrgicos}

Foram utilizados 20 cães adultos, 16 machos e 4 fêmeas, sem raça definida, pesando entre 8,6 e 17,2 quilos (média de 12,98 quilos). Após exames clínicos e neurológicos e da verificação de ausência de alterações que pudessem interferir com o experimento, os animais foram alojados em duplas, em canis. Vinte e quatro horas antes da cirurgia os cães foram pesados e submetidos à tricotomia da área cirúrgica. O regime anestésico utilizado, consistiu em pré-medicação com sulfato de atropina $(0,05 \mathrm{mg} / \mathrm{kg})$, seguida 15 minutos após, da administração de cloridrato de xilazina $(1 \mathrm{mg} / \mathrm{kg})$, ambos pela via subcutânea; seguidos de tiopental sódico (aproximadamente 12,5mg/kg), pela via intravenosa, até o terceiro estágio de Guedel. Usaram-se enrofloxacina $(5 \mathrm{mg} / \mathrm{kg})$ pela via subcutânea, duas horas antes do ato cirúrgico e a cada 24 horas após, durante 5 dias, e, cefalotina sódica $(25 \mathrm{mg} / \mathrm{kg})$, endovenosa, 30 minutos antes e duas horas após a incisão da pele.

As abordagens dorsolateral esquerda, para os processos espinhosos e corpos vertebrais de $\mathrm{L}_{1}$ até $\mathrm{L}_{4}$ inclusive, e dorsolateral direita, para os processos espinhosos e lâminas das vértebras $L_{1}$ até $\mathrm{L}_{4}$ inclusive, seguiram técnica descrita por TUDURY (1985). Os vasos e o nervo espinhal da face lateral esquerda do forame intervertebral $L_{2}-L_{3}$ foram seccionados e ligados. A junção intervertebral $\mathrm{L}_{2}$ $\mathrm{L}_{3}$ foi desestabilizada, seccionando-se com lâmina de bisturi $\mathrm{n}^{\circ} 11$, os $4 / 5$ (quatro quintos) ventrais do disco intervertebral.

Inicialmente, fizeram-se quatro perfurações, na face dorso-lateral esquerda dos corpos vertebrais $\mathrm{L}_{2}$ - $\mathrm{L}_{3}$, no sentido ventro-lateral oposto, com broca ortopédica e furadeira manual, seguindo-se o emprego do macho ortopédico para a feitura das roscas. O diâmetro do macho e dos parafusos ortopédicos de aço 
inoxidável empregados foi preestabelecido, quando da confecção das placas, ficando em 3,5 ou 4,5mm. $\mathrm{O}$ diâmetro das brocas, para cada tipo de parafuso, foi de 2,5 e $3,2 \mathrm{~mm}$, respectivamente. O comprimento dos parafusos foi determinado no transoperatório, com medidor de profundidade, de tal maneira que sua extremidade sobressaísse no mínimo $5 \mathrm{~mm}$ da superfície vertebral. A placa foi adaptada aos corpos vertebrais L; e L3 e fixada através de quatro parafusos, sendo dois em cada corpo vertebral Imediatamente após a colocação firme dos parafusos e da placa (Figura 1), testou-se a estabilidade vertebral e se desestabilizou o restante dessa articulação intervertebral, realizando-se a remoção bilateral dos processos articulares e secção dos ligamentos amarelo, interespinhoso e supraespinhoso.

Em ambos os lados dos processos espinhosos das vértebras $\mathrm{L}$ ), L;, 1.3 e 1.4, e bem próximo da sua base, foram colocadas as placas arciformes, fixadas com fio ortopédico de aço inoxidável, de $1 \mathrm{~mm}$ de diâmetro, passados através dos espaços interespinhosos (Figura 1). Nos espaços Ti3$\mathrm{Li}$ e 1.4-1.5, cada fio passou pêlos orifícios das placas, sendo amarrado até se justaporem suas extremidades. Nos espaços Li-L; 1.2-1.3 e L3-L.4, o fio circundou as placas, aproximando-as, ao ser torcido.

\section{Imediatamente}

após a fixação das placas nos processo espinhosos, testou-se a estabilidade das cerciagens e das vértebras, seguindo-se irrigação dos tecidos expostos com 500 $\mathrm{ml}$ de soro fisiológico estéril. A síntese de todos os planos cirúrgicos foi realizada com fio de nylon, monofilamento $\mathrm{n}^{\circ}$ 2-0. Utilizaram-se pontos contínuos festonados, na faseia toracolombar externa profunda; pontos contínuos simples, no tecido subcutâneo, músculo cutâneo e faseia toracolombar externa superficial e pontos simples separados, na pele.
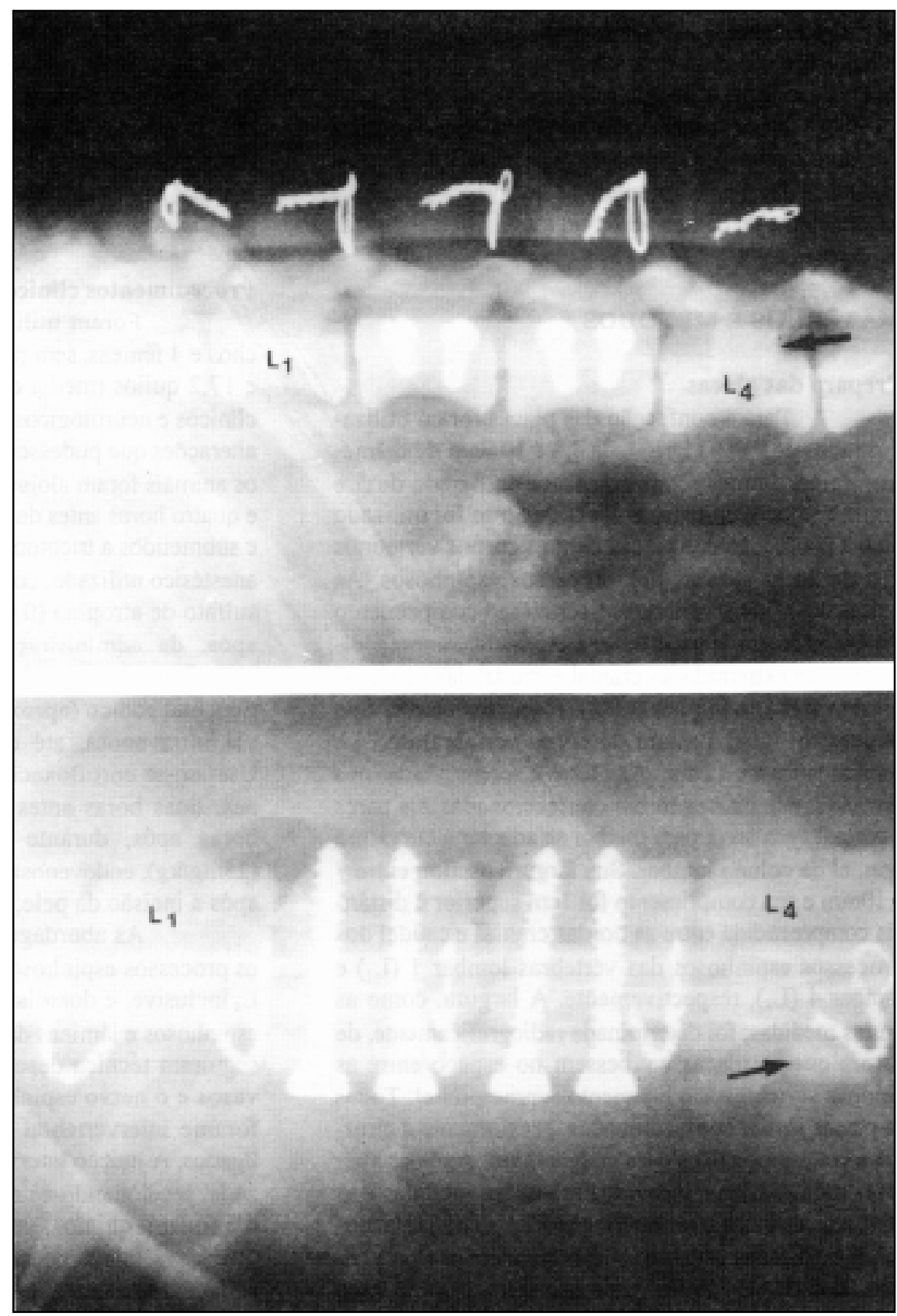

Figura 1 - Radiografias lateral e ventro-dorsal da coluna vertebral lombar de cão. Placa de PVC fixada com 4 parafusos nos corpos vertebrais $\mathrm{L}_{2}$ e $\mathrm{L}_{3}$ e duas placas, fixadas com fio ortopédico de aço inoxidável, nos processos espinhosos de $\mathrm{L}_{1}, \mathrm{~L}_{2}, \mathrm{~L}_{3}$ e $\mathrm{L}_{4}$. Fragmento de PVC apoiado na face lateral $\mathrm{L}_{4}$, para satisfazer análise de outra pessoa. 
Durante cinco dias manteve-se bandagem compressiva no local.

Análises radiográficas da coluna vertebral lombar, nas incidências lateral e ventrodorsal, foram realizadas imediatamente após a cirurgia e aos 15 e 45 dias depois. Aos 45 dias do pós-operatório, após as avaliações clínicas, os animais foram sacrificados. À necropsia foram analisadas as alterações macroscópicas dos tecidos circunjacentes à área operada, a estabilidade vertebral (com os implantes e após remoção deles), a ocorrência de alterações e enfraquecimento dos materiais implantados, o dano às estruturas ósseas, o afrouxamento ou ruptura dos implantes e lesões orgânicas sugestivas de rejeição ao material implantado.

Previamente às cirurgias, assim como, 15 e 45 dias após à implantação das placas, os cães foram submetidos à colheita de sangue e urina para determinação do eritrograma, leucograma, número de plaquetas, urinálise e dosagem da enzima alanino-aminotransferase, albumina, bilirrubinas e creatinina séricas.

A variável peso dos animais, dos dias $\mathrm{O}$, 15 e 45, foi analisada mediante esquema de comparação de pares, utilizando-se o teste "T" de Student para a comparação das médias (SNEDECOR \& COCKRAN, 1989). As variáveis peso dos animais e a distância: borda da placa/perímetro do orifício, dos grupos com e sem rompimento das placas dos corpos vertebrais, foram submetidas à análise da variância, sendo as médias comparadas pelo teste "T" de Student. A relação peso dos animais/rompimento de placas foi também analisada através do teste exato de Fisher (GOMES, 1976), sendo os animais classificados como leves ou pesados, utilizando-se como base, a média de peso dos mesmos, no dia da necropsia.

\section{RESULTADOS}

\section{Observações clínicas}

O PVC mostrou-se fácil de ser manipulado (cortado, perfurado, polido, lavado e esterilizado) e economicamente viável. A esterilização por calor não deformou nem mudou a cor das placas, e aparentemente, não modificou sua rigidez e resistência. A flexibilidade do plástico permitiu íntimo contato entre a placa e os corpos vertebrais, bem como aproximação adequada entre as placas de $2 \mathrm{~mm}$, através das cerciagens, comprimindo e envolvendo cada um dos processos espinhosos. As placas proporcionaram alinhamento espinhal restabelecendo a estabilidade e a rigidez vertebrais.

Nenhum animal exibiu infecção ou deiscência de ferida. A temperatura corporal oscilou entre
39,3 a $40,5^{\circ} \mathrm{C}$, em 14 dos 20 animais, nas primeiras 24 e até às 96 horas após a colocação das placas. Sinais de dor foram constatados à deambulação e ao alçar os animais, mas apenas, durante os primeiros 3 a 5 dias. Após 15 dias, todos os animais exibiram movimentação normal, chegando a correr, pular e a se erguerem apoiados apenas nos membros posteriores.

Nenhum dos cães exibiu sinais compatíveis com trauma da medula espinhal ou falha dos implantes. Também não se observou deslizamento dorsal das placas arciformes ou presença de seromas. A coluna toracolombar manteve-se sempre alinhada e somente quatro cães permaneceram com discreta cifose até o final do experimento. Paralisia muscular da parede abdominal esquerda foi constatada em 11 animais, perdurando pêlos 45 dias, em 10 deles. Os animais pesaram 13,35 $\pm 2,59 ; 13,31 \pm 2,40$ e $13,54 \pm 2,67 \mathrm{~kg}$ nos dias O (prévio), 15 e 45 da cirurgia vertebral, sendo que pela análise pareada constatou-se diferença significativa $(\mathrm{P}<0,01)$ entre os dias $\mathrm{O}$ e 45.

Nas análises radiográficas, a coluna vertebral toracolombar permaneceu normal e com aspecto idêntico ao inicial. As placas fixadas nos corpos e processos espinhosos vertebrais mantiveram a coluna alinhada em todas as imagens radiográficas. No exame radiológico imediato à cirurgia, foi verificado discreto afastamento da placa fixada nos corpos vertebrais, em um único animal. Distanciamento idêntico, foi constatado nesse e noutro animal, aos 45 dias. Numerando os parafusos colocados nos corpos vertebrais, no sentido cranial para o caudal como 1, II, III e IV, verificou-se, aos 45 dias, migração parcial dos parafusos I, II e III num desses cães e do II noutro animal. Em nenhum caso foi vista reação periosteal na área de contato com o PVC. Embora não tenha sido vista, radiograficamente, ruptura de placas arciformes e cerciagens, rompimento de placas dos corpos vertebrais foi visualizado em três animais, aos 45 dias.

O resultado dos exames laboratoriais, assim como, a comparação dos valores obtidos nos diferentes períodos de colheita, não evidenciou toxicidade do material.

\section{Observações à necropsia}

Tanto nos processos espinhosos como nos corpos vertebrais as placas estavam firmemente envolvidas por cápsula fibrosa. Os orifícios da placa fixada nos corpos de $\mathrm{L}^{\wedge}$ e 1.3 mostravam início de invasão por tecido fibroso, mas não se visualizou nenhum tipo de secreção nas interfaces vértebraplaca e placa-cápsula fibrosa. Reação periosteal, proliferação ou alteração óssea estavam ausentes e não foi detectada nenhuma mudança de cor ou forma das 
placas. Em todos os casos, o segmento vertebral cirurgicamente enfraquecido, com e após a remoção das placas, mostrava-se alinhado e perfeitamente estável. Nenhum dos processos espinhosos imobilizados mostrava sinais de fratura.

Apesar da migração parcial de parafusos, em dois animais, os mesmos mantinham-se ainda firmemente fixados. Nenhum dos fios ortopédicos ou parafusos exibiu sinais de desgaste, enfraquecimento, deterioração ou rompimento. Nenhuma das placas arciformes exibiu fissuras ou rupturas, apenas as marcas superficiais deixadas pelas cerciagens. A ruptura da placa fixada no corpo vertebral ocorreu em oito animais. Sempre transversal e nos orifícios II e/ou III, próximos ao disco intervertebral. Em três dos quatro animais com cifose, verificou-se à necropsia, fissura dorsal da placa, em um dos orifícios centrais. Em todos os casos, o tecido fibroso que envolvia a placa, mantinha os fragmentos do PVC em intimo contato (sem a existência de uma fenda). Os resultados da análise da variância e os valores médios do peso dos animais e da distância média entre a borda da placa e o perímetro do orifício, nos animais com e sem ruptura da placa, constam na Tabela 1. Dos oito animais nos quais a placa sofreu ruptura, quatro foram classificados como pesados. Dos outros 12 animais, cujas placas não sofreram fratura, oito foram classificados como leves. Aplicando-se o teste de Fisher (GOMES, 1976) a estes resultados, ratificou-se que o peso dos animais, dentro do intervalo estudado $(8,5$ a $18,7 \mathrm{~kg})$, não exerceu influência significativa $(\mathrm{p}>0,05)$ na fratura das placas.

Tabela 1 - Valor médio, desvio padrão e resultado da análise estatística do peso dos animais e da distância: borda da placa-perímetro do orifício, nos grupos com e sem rompimento da placa dos corpos vertebrais.

\begin{tabular}{lcc}
\hline $\begin{array}{l}\text { Parâmetro } \\
\text { Grupo }\end{array}$ & Peso corporal (kg) & $\begin{array}{c}\text { Distância BP-PO } \\
(\mathrm{mm})\end{array}$ \\
\hline Com rompimento & $14,800^{\mathrm{a}} \pm 2,694$ & $3,093^{\mathrm{a}} \pm 0,431$ \\
& $12,691^{\mathrm{a}} \pm 2,394$ & $2,906^{\mathrm{a}} \pm 0,532$ \\
\hline
\end{tabular}

Médias da mesma coluna, seguidas por letras iguais, não diferem estatísticamente $(\mathrm{p} \geq 0,05)$ entre $\mathrm{si} ; \mathrm{BP}-\mathrm{PO}=$ borda da placa perímetro do orifício.

\section{DISCUSSÃO E CONCLUSÕES}

A inovação pretendida com esta pesquisa foi o uso de placas ortopédicas feitas de PVC, na imobilização de trataras e luxações da coluna vertebral de cães. ALMEIDA \& REZENDE (1990), REZENDE \& ALMEIDA (1990) e RODRIGUEZ ALVAREZ (1990) já empregaram em ossos longos, placas feitas com esse material. Como as fraturas e luxações instáveis da espinha devem ser tratadas por métodos de imobilização interna (BRUECKER \& SEIM, 1993) e como, em geral, as placas ortopédicas são indicadas para estabilização de estruturas ósseas (De YOUNG \& PROBST, 1993), resolveu-se, baseado nas vantagens apresentadas pelas placas de PVC, quando usadas em ossos longos (ALMEIDA \& REZENDE, 1990; REZENDE \& ALMEIDA, 1990; RODRIGUEZ ALVAREZ, 1990), testa-las em traumatologia espinhal.

Preferiu-se, nesta pesquisa, fixar as placas plásticas, tanto nos corpos como nos processos espinhosos vertebrais, visto que WALTER $\boldsymbol{e t}$ al. (1986) e WALKER (1990) afirmaram que, frente às forças de flexão, esse seria o meio mais seguro de imobilizar a coluna vertebral. A dúvida era se as placas de PVC poderiam substituir as metálicas e as Lubra, usadas na cirurgia da coluna vertebral (BRUECKER \& SEIM, 1993). As mesmas características de flexibilidade, facilidade de corte e modelagem das Lubra (YTURRASPE \& LUMB, 1972; RENEGAR, 1990) foram vistas no PVC, com a vantagem do custo mais baixo. Outra característica apreciável foi a de se conseguir, com o PVC, placas de superfícies lisas que, segundo HOERLEIN (1978) diminuiria os riscos de infecção.

O uso de placas plásticas, tanto nos corpos como nos processos espinhosos vertebrais, já foi recomendado por YTURRASPE \& LUMB (1973) e WALKER et al. (1985b). A diferença está no material, PVC em substituição ao fluoreto de polivinilideno. A imobilização de apenas dois processos espinhosos, cranial e caudal ao local de instabilidade, conforme sugestão de RENEGAR (1990), evitou de fato, o enrijecimento de um grande segmento espinhal, diminuindo provavelmente o estresse sobre as placas arciformes. Também contou-se com a complementação da imobilização proporcionada pela placa fixada nos corpos vertebrais, para se conseguir suficiente estabilidade. Por outro lado, a fixação tão só, dos dois processos espinhosos próximos à luxação, possivelmente não teria sido suficiente para se conseguir estabilização segura. As placas de PVC, por serem plásticas, não sofreriam a corrosão natural das metálicas (KELLEY et al., 1987), sendo que, poderiam reduzir a corrosão eletrolítica dos parafusos (efeito isolante). 
Quarenta por cento das placas colocadas nos corpos vertebrais romperam-se. Somando as rupturas observadas por RODRIGUEZ ALVAREZ (1990) e" as ocorridas nesta pesquisa, parece que o PVC-Tigre ${ }^{\circ}$ não apresenta resistência suficientemente adequada, para uso em ortopedia. $\mathrm{O}$ rompimento não se deveu a falha de técnica, já que não ocorreu nos cães onde as placas ficaram afastadas nem naqueles com migração dos parafusos. A ruptura pode, então, ter ocorrido pela atuação de forças de flexão e/ou torção sobre o material, num período tardio (quando os animais já se movimentavam à vontade). WALDRON et $\boldsymbol{a l}$. (1991) já haviam constatado fraqueza do método de imobilização usado nesta pesquisa, frente aos movimentos de torção. Os resultados da análise estatística, nesta pesquisa, não confirmaram também, qualquer relação entre a ruptura da placa e a distância do orifício à borda da placa, bem como, entre rompinmento do plástico e peso dos cães (dentro do intervalo 8,5 a $18,7 \mathrm{~kg}$ ).

Outro fator que pode ter influenciado a ruptura das placas é sua espessura. As usadas neste trabalho foram, respectivamente, 1,5 e 2,0mm, mais espessas que as utilizadas por RODRIGUEZ ALVAREZ (1990) e as placas de fluoreto de polivinilideno (SWAIM et al., 1988). A maior espessura não impediu a fratura do implante, levando a crer que a falha se deveu à resistência do PVC. O diâmetro do parafuso de fixação tem que ser levado em conta. RODRIGUEZ ALVAREZ (1990) fixou as placas de PVC, no rádio, com parafusos corticais de $3,5 \mathrm{~mm}$. Entretanto, a literatura consultada não foi clara com relação ao diâmetro dos parafusos a serem usados nas vértebras. Com base no tipo de placas usadas, na razão parafüsosplacas e parafüsos-corpos vertebrais e no diâmetro das brocas, foi deduzido que o diâmetro dos parafusos utilizados por outros cirurgiões oscilava entre 2,0 a 4,5 mm (SWAIM, 1971; WALKER $\boldsymbol{e t}$ al., 1985b; WALTER et al., 1986). Devido a isto, optou-se por parafusos de $4,5 \mathrm{~mm}$, para as placas de $12 \mathrm{~mm}$ de largura, e de $3,5 \mathrm{~mm}$, para as mais estreitas. Como romperam, duas das três placas nas quais foram usados parafusos de $4,5 \mathrm{~mm}$, poderia ser o diâmetro do parafuso um fator importante no desencadeamento deste acontecimento. Mas, nas outras seis placas rompidas foram usados parafusos de $3,5 \mathrm{~mm}$.

Como não foi possível definir claramente a causa da ruptura das placas, sugere-se fazer a imobilização externa adicional, durante 45 a 60 dias, como aconselha a literatura (RENEGAR, 1990). Também poderia empregar-se placas mais largas, mais espessas e/ou parafusos com diâmetro inferior a $3,5 \mathrm{~mm}$, bem como placas com espessura variável conforme o peso dos animais.

O rompimento das placas dos corpos vertebrais deve ter ocorrido entre 15 a 44 dias após sua fixação. Com certeza, foi posterior à cicatrização fibrosa estável das estruturas vertebrais, isto porque nenhum dos oito cães apresentou sinais clínicos, neurológicos e/ou radiológicos de instabilidade; a solução de continuidade das placas só foi observada nas radiografias do $45^{\circ}$ dia e, à necropsia, os fragmen- tos da placa mantinham-se em íntimo contato (sem a existência de fenda), pela ação da cápsula fibrosa. A dificuldade de se visualizar radiografícamente, a fragmentação das placas, decorreu da imobilização das partes pela fibrose, e da superposição dos parafusos.

A não ocorrência de rompimento do fio ortopédico, das placas arciformes e dos processos espinhosos; bem como ausência de seromas e deslizamentos dessas placas, parece assinalar que a técnica foi efetiva. Por indicação de BRASMER \& LUMB (1972) estas placas foram feitas em forma de arco, acompanhando a curvatura normal da coluna vertebral. Duas modificações foram então introduzidas nesta técnica - o uso de placas de PVC e - o uso de fio ortopédico entre os processos espinhosos e não através deles. Esta modificação da técnica, diferindo portanto de MÜLLER (1991), baseou-se no fato de que a irrigação desses processos realiza-se por via intraóssea, a partir da lâmina vertebral (RISCHEN et al., 1987; ALBERTI \& PINA, 1989) e que lesões vasculares poderiam predispor ao enfraquecimento e à fratura dos mesmos (RISCHEN et al., 1987). Como mencionado por YTURRASPE \& LUMB (1973) e WALKER et al. (1985b), a presença de placas nos corpos vertebrais pode ter contribuído para a ausência de fratura dos processos espinhosos. Mas, considerando-se o rompimento de $40 \%$ das placas dos corpos vertebrais e nenhuma ruptura naquelas empregadas nos processos espinhos, poder-se-ia dizer que o método utilizado nestes últimos foi eficiente. O PVC de $2 \mathrm{~mm}$ mostrou ter flexibilidade suficientemente para, sem quebrar, envolver, um a um, cada processo espinhoso, sem os afrouxamentos ou deslocamentos, citados por WALKER (1990). O uso de fio ortopédico, em sustituição aos parafusos, teve objetivo económico e baseou-se nas sugestões de HOERLEIN (1978) e MÜLLER (1 991).

\section{FONTES DE AQUISIÇÃO}

a - Tubos Marca Tigre: Companhia Hansen Industrial, Rua Xavantes, 54 Joinville, SC. (89203-210). Brasil. 


\section{REFERÊNCIAS BIBLIOGRÁFICAS}

ALBERTI, A., PINA, J.A.E. Diaphanisation et étude vasculaire de Ia vertèbre de chien. Bui Assoe Anat, Nancy, v. 73, p. 5-7, 1989.

ALMEIDA, A.E.R., REZENDE, C.M. de F. Confecção de placas ortopédicas de cloreto de polivinila para redução de fraturas de ossos longos do cão. In: CONGRESSO MINEIRO DE MEDICINA VETERINÁRIA, 4, 1990, Belo Horizonte, MG. Anais... Belo Horizonte: Escola de Veterinária da UFMG, 1990. p. 82,287 p.

BRASMER, T.H., LUMB, W.V. Lumbar vertebral prosthesis in the dog. Am J Vet Rés, Schaumburg, v. 33, n. 1, p. 493-500, 1972 .

BRASMER, T.H. The acutely traumatized small animal patient. Philadelphia: Saunders, 1984. 167 p.

BRINKER, W.O., PIERMATTEI, D.L. , FLO, G.L. Handbook of small animal orthopedics $\&$ fracture treatment. 2 . ed. Philadelphia: Saunders, 1990. 582 p.

BRUECKER, K..A., SE1M III, H.B. Spinal fractures and luxations. In: SLATTER, D. Textbook of small animal surgery. 2. ed. Philadelphia: Saunders, 1993. Cap. 78, p. $1110-1121$.

CERVERA, M., DOLZ, M., HERRAEZ, J.V., et al. Evaluation of the elastic behaviour of central venous PVC, polyurethane and silicone catheters. Phys Med Biol, London, v. 34, n. 2, p. 177-183,1989.

DEMERTZIS, P., KONTOMINAS, M.G. Study of sorption of vinylchioride on unplasticized polyvinylchioride in model food systems by clássica! partition: Effect ofmonomer concentration, temperature and polymer particle size. Lebensmittel-Wissenschaft und Technologie, Kuesnacht, v. 19, n. 1, p. 1-6, 1986.

DeYOUNG, D.J., PROBST, C.W. Methods of internai fracture fixation. General principies. In: SLATTER, D. Textbook of small animal surgery. 2. ed. Philadelphia: Saunders, 1993. Cap. 123 , p. $1610-1631$.

FIGGE, K., KOCH, J., FREYTAG, W. The suitability ofsimulants for foodstuffs, cosmetics and pharmaceutical products in migration studies. Food Cosmet Toxicol, Oxford, v. 16 , p. $135-142,1978$

GERHARDS, H., RADICKE, S., HIPP, K.P. Aniage, Pflege und Nutzung von Dünndannfisteln bei Ponys. Pferdeheilkunde, Neubulach, v. 7, n. 5, p. 243-248, Sept./Oct. 1991

GOMES, F.P. Curso de estatística experimental. 6 ed. São Paulo: Nobel, 1976. 430 p.

GOTTESMAN, R.T., GOODMAN, D. Poly (vynil) chioride. In: TESS, R.W., POEHLEIN, G.W. Appiied polymer science. 2. ed. Washington: American Chemical Society, 1985. cap. 18 , p. $383-440$

GOWARIKER, V.R., VISWANATHAN, N.V., SREEDHAR, J. J. Polymer science. New York: John Wiley \& Sons, 1986. $505 \mathrm{p}$.

HAYHURST, E.G., WYMAN, M. Morbidity associated with prolonged use of polyvinyl fedding tubes. Am J Dis Child, Chicago, v. 129, p. 72-74, 1975.
HOERLEIN, B.F. Canine neurology. Diagnosis and treatment. 3. ed. Philadelphia: Saunders, 1978. 791 p.

HORNE, R.D. The use ofmodem bone-plating equipment in small animal orthopedics. Vet Med Small Anim Clin, Edwardsville, v. 66, n.6,p.576-584, 1971.

HUIBREGTSE, K. Plastic or expandable biliary endoprotheses? Scandinavian J Gastroenterol, Toyen, v. 28, n. 200, p. 3-7, 1993.

JANISCH, H., MICHALICA, W., PICHA, E., et al. Mukopyometra und Korpuskarzinom. Strahientherapie, Munich, v. 143 , n. 5, p. 511-515,1972

JOHNSON, W.S., SCHMIDT, R.E. Effects of polyvinyl chioride ingestion by dogs. Am J Vet Rés, Schaumburg, v. 38, n. 11, p. 1891-1892, 1977.

KELLEY, B.S., DUNN, R.L., CASPER, R.A. Totally resorbable high-strength composite material. In: GEBELEIN, C.G. Advances in biomedical polymers. New York: Plennum, 1987. p. $75-85$

KEMP, C., K.RUGER, J.S.J. Atrial cannulation for long term sequential blood coilection. Am J Vet Rés, Schaumburg, v. 48, n. 6, p. 990-991, 1987.

KONTOMINAS, M.G., DEMERTZIS, P.G., GILBERT, S.G. Sorption of vinylchioride into polyvinylchioride by classical partition and inverse gás chromatography: Comparison of two methods. J Food Process Preserv, Westport, v. 9, n. 4, p. 223-234,1985.

Mc. KEE, W.M. Spinal trauma in dogs and cats: A review of51 cases. Vet Rec, London, v. 126, n. 6, p. 285-289, 1990.

MILLER, W.H., MALTBY, M. A rumen cannula for small ruminants. J Range Manage, Denver, v. 39, n. 5, p. 474-475, 1986.

MÜLLER, D. Lubraplattentechnik zur Stabilisierung von Wirbelsaulenfrakturen. Praktische Tierarzt, Hannover, v. 72, n. 9 , p. $729-732,1991$

PAWDE, A.M., MARUDWAR, S.S. Peritoneal dialysis in experimental peritonitis in calves. Indian Vet J, Madras, v. 70, n. 7, p.645-647,1993.

RENEGAR, W.R. Axial skeletal fractures. In: WHITTICK, W.G. Canine orthopedics. 2. ed. Philadelphia: Lea \& Febiger, 1990. Cap. 14, p. 308-356.

REZENDE, C.M. de F., ALMEIDA, A.E.R. Emprego de placas de cloreto de polivinila no tratamento de fraturas. In CONGRESSO MINEIRO DE MEDICINA VETERINÁRIA, 4, 1990, Belo Horizonte, MG Anais... Belo Horizonte, Escola de Veterinária da UFMG, 1990. p. 83,287 p.

RISCHEN, C.G., WILSON, J.W., SWAIM, C.A. Effect ofappiication of polyvinilidine plates on the dorsal spinous process of dogs. Vet Surg, Philadelphia, v. 16, n. 4, p. 294 298, 1987.

RODRIGUEZ ALVAREZ, L.M.R. Emprego de placas de cloreto de polivinila na fixação de fratura do terco distai do rádio e uina no cio novo (Canis familiaris). Estudo experimental. Belo Horizonte - MG. 44 p. Tese (Mestrado Medicina Veterinária) - Escola de Veterinária, Universidade Federal de Minas Gerais, 1990. 
SABADEANU, V., POPA, P.D., MULFAY, V, Une methode nouvelle de fixation de Ia prothese lacrymale. Arch Ophthalol, Paris, v. 32, n. 2, p. 151-154, 1972.

SMITH, G.K., WALTER, M.C. Fractures and luxations of the spine. In: NEWTON, C.D.; NUNAMAKER, D.M. Textbook of small animal orthopaedics. Philadelphia: Lippincott, 1985. cap. 19, p. 307-332.

SNEDECOR, G.W., COCKRAN, W.G. Statistical methods. 8 ed. lowa: lowa State University Press, 1984. 503 p.

SWAIM, S.F. Vertebral body plating for spinal imobilization. J Am Vet Med Assoe, Schaumburg, v. 158, n. 10, p. 16831693, May 1971.

SWAIM, S.F. Vertebral and spinal cord surgery. In: OLIVER, J.E., HOERLEIN, B.F., MAYHEW, I.G. Veterinary neurology. Philadelphia: Saunders, 1987. Cap. 17, p, 416469.

SWAIM, S.F., RIBBLE, G.A., WILSON, J.W. Histologic evaluation of response to implantation ofpolyvinilidene plates in dogs and rats. Vet Surg, Philadelphia, v. 17, n. 5, p. 278 $281,1988$.

TUDURY, E.A. Comparação das abordagens dorsolateral e lateral na região toracolombar, para hemilaminectomia. durotomia e fenestração terapêutica e profilática à protrusâo dos discos intervertebrais em cies sadios. Santa Maria - RS. 113 p. Dissertação (Cirurgia Veterinária) - Curso de Pós-graduação em Medicina Veterinária, Universidade Federal de Santa Maria,-1985.

TUDURY, E.A., FIALHO, S.A.G., PAULO, N.M., $\boldsymbol{e} \boldsymbol{t} \boldsymbol{a l}$ Avaliação de dois métodos para prevenção da cifose pósoperatória na laminectomia dorsal de cães. Rev Centro Ciênc. Rurais, Santa Maria, v. 15, n. 1, p. 57-68, 1985.

TURNER, W.D. Fractures and fractures-luxations of the lumbar spine: a retrospective study in the dog. J Am Anim Hosp Assoe, Denver, v. 23, p. 459-464, July/Aug. 1987.

VASILEV, D., VLASOVA, D., FILIPOV, S., et al. Our experience with the use of the new Bulgarian intrauterine contraceptive device, the Vénus $\mathrm{Cu}$ 300. Akush Ginekol, Sofiia, v. 30, n. 2, p. 39-43, 1991

VINYL CHLORIDE, polyvinyl chioride and vinyl cnloride-vinyl acetato copolymers. Monogr Eval Carcinog Risk Chem Hum, Lyons,v. 19, p. 377-438, 1979.

WALDRON, D.R., SHIRES, P.K., Me CAIN, W., et al. The rotational stabilizing effect of spinal fixation techniques in an unstable vertebral model. Prog Vet Nerol, Washington, v. 2, n. 2, p. 105-110, 1991.

WALKER, J.W., STUTH, J.W., HEITSCHMIDT, R.K., et al. A new esophagal plug. J Range Monage, Denver, v. 38, n. 2, p. 185-187, Mar. 1985a.

WALKER, T.L., TOMLINSON, J., SORJONEM, D.C., $\boldsymbol{e} \boldsymbol{t}$ al. Disease ofthe spinal column. In: SLATTER, D.H. Textbook of small animal surgery. Philadelphia: Saunders, 1985b. Cap. 97, p.1367-1395.

WALKER, T.L. The thoracolumbar spine. In: HARVEY, C.E., NEWTON, C.D., SCWARTZ, A. Small animal surgery. Philadelphia: Lippincott, 1990. Cap. 19. p. 514-531.

WALTER, M.C., SMITH, G.K., NEWTON, C.D. Canine lumbar spinal internai fixation techniques. A comparative biomechanical study. Vet Surg, Philadelphia, v. 15, n. 2, p. 191-198, 1986

WILLIAMSON, ]., KAVANAGH, B. Vinyl chioride monomer and other contaminants in PVC welding fume. Am.Ind Assoe J, New York, v. 48, n. 5, p. 432-436, 1987.

YTURRASPE, D.J, LUMB, W.V. The use ofplastic spinal plates for internai fixation of the canine spine. J Amer Vet Med Assoe, Schaumburg, v. 161, n. 12, p. 1651-1657, Dec. 1972.

YTURRASPE, D.J., LUMB, W.V. Second lumbar spondylectomy and shortening ofthe spinal column ofthe dog. Am J Vet Rés, Schaumburg, v. 34, n. 4, p. 521-525, Apr., 1973. 